\title{
Combined 5G-Based Video Production and Distribution in a Crowded Stadium Event
}

\author{
Ioannis P. Chochliouros $^{1\left(\mathbb{}{ }^{(}\right)}(\mathbb{D})$, Anastasia S. Spiliopoulou ${ }^{1}$, \\ Pavlos Lazaridis ${ }^{2}$, Michail-Alexandros Kourtis ${ }^{3}$, \\ Zaharias Zaharis ${ }^{4}$, and Alexandros Kostopoulos ${ }^{1}$ \\ ${ }^{1}$ Hellenic Telecommunications Organization (OTE) S.A., 99 Kifissias Avenue, \\ 15124 Maroussi-Athens, Greece \\ \{ichochliouros, alexkosto\}@oteresearch.gr, \\ aspiliopoul@ote.gr \\ 2 The University of Huddersfield, Queensgate, Huddersfield HD13DH, UK \\ P. Lazaridis@hud.ac.uk \\ 3 National Centre for Scientific Research "Demokritos", \\ 15310 Aghia Paraskevi-Athens, Greece \\ akis.kourtis@iit.demokritos.gr \\ 4 Aristotle University of Thessaloniki, 54124 Thessaloniki, Greece \\ zaharis@auth.gr
}

\begin{abstract}
Based upon the scope of the original 5G ESSENCE research effort and by considering the related fundamental architecture, we develop a dedicated scenario for the implementation and demonstration of a setup for a $5 \mathrm{G}$ edge network acceleration in the context of a sport event, taking place in a stadium. Specifically, we demonstrate a combined 5G-based video production and video distribution scenario towards delivering benefits to both media producers/content providers and mobile operators, with those being able to offer enriched event experience to their subscribers. The production/distribution of locally generated content through the respective platform, coupled with value-added services and rich user context, enables secure, high-quality and resilient transmission in realtime, thus ensuring minimal latency. In the selected scenario, massive data traffic does not affect nor overload the backhaul connection as it is produced, processed and consumed just locally, aligned to the 5G 3GPP specifications. We also discuss details about the proposed services and functionalities of the use case, together with an approach for a potential projection to the market.
\end{abstract}

Keywords: $5 \mathrm{G} \cdot$ Cloud-Enabled Small Cell $(\mathrm{CESC}) \cdot$ evolved Multimedia Broadcast Multicast Services (eMBMS) $\cdot$ Multi-access Edge Computing $(\mathrm{MEC}) \cdot$ Network Functions Virtualisation $(\mathrm{NFV}) \cdot$ Small Cell $(\mathrm{SC}) \cdot$ Virtual Network Function (VNF)

\section{Introduction}

The introduction of 5G technologies in economic and societal procedures of everyday life is a "key" asset that supports transformation of the vertical industries and focuses upon the development of cloud infrastructure and the concomitant development of 
mobile broadband digital access networks $[1,2]$. The advent of the Internet of Things (IoT) and the predictive and real-time intelligence on network devices act as a "catalyst" to the growing adoption of edge cloud solutions and services. To respond, the next generation of communication and services includes virtualised applications that "run" much closer to mobile users ensuring network flexibility, economy and scalability [3]. The delivery of services from an edge cloud represents a new synergistic business model between network service providers or edge cloud providers and enterprises [4]. For the edge cloud provider, it represents an opportunity to nearly double the revenues associated with the new services, while for an Information Communication Technology (ICT) services enterprise it shows a potential opportunity to "add" a new revenue stream to its portfolio by using and delivering over the top (OTT) services [5].

The 5G ESSENCE project [6] addresses the paradigms of Multi-access Edge Computing (MEC) and Small Cell as-a-Service (SCaaS) [7] by fueling the drivers and removing the barriers in the Small Cell (SC) market, forecasted to grow at an impressive pace up to 2020 and beyond and to play a "key" role in the 5G ecosystem. The proposed approach provides a highly flexible and scalable platform, which is able to support new business models and revenue streams by creating a neutral host market and reducing operational costs by providing new opportunities for ownership, deployment, operation and amortisation [8].

Among other potential applications and within all proposed use cases, this scope delivers benefits to media producers and mobile operators, enabling them to offer a highly interactive fan experience and to optimise operations by deploying key functionalities at the edge (i.e., evolved Multimedia Broadcast Multicast Services (eMBMS) together with multitenancy support from small cells). By leveraging the benefits of small cell virtualisation and radio resource abstraction, as well as by optimising network embedded cloud, it becomes possible to "ease" the coverage and capacity pressure on the multimedia infrastructure, and also to increase security since content will remain locally. Furthermore, additional benefits for the operators and the venue owners arise such as: (i) Lower latency, due to shortening the data transmission path, and; (ii) increased backhaul capacity, due to playing out the live feeds and replays locally, that practically "puts" no additional strain on the backhaul network and upstream core network components. Among the fundamental 5G ESSENCE use cases [9] is the one about supporting 5G edge network acceleration in a crowded event, that is discussed and further assessed in the continuity of the present work.

\section{Basic Architecture and Use Case Description}

The 5G ESSENCE project realises a two-tier cloud architecture that enables the provision of dynamically repurposed virtual network infrastructures with tailored computing and flexible networking capabilities. Following the related approach, the Small Cell concept is evolved as not only to provide multi-operator radio access, but also to achieve an increase in the capacity and the performance of current RAN (Radio Access Network) infrastructures, and to extend the range of the provided services while maintaining its agility [10]. To achieve these ambitious goals, our scope provides an enhanced, edge-based, 
virtualised execution environment attached to the small cell, taking advantage and reinforcing the concepts of MEC [11] and network slicing [12, 13]. The existing 5G architecture is a solid reference point for our original approach, which combines the current 3GPP framework to network management in RAN sharing scenarios [14] and the ETSI NFV framework for managing virtualised network functions [15].

The CESC (Cloud-Enabled Small Cell) provides the computing, storage and radio resources at the edge, while the CESC cluster is the cloud platform. This cloud platform can also be "sliced" to enable multi-tenancy, and it is used to support Virtualised Network Function (VNFs) implementing the different features of the SCs as well as to support the mobile edge applications delivered to the end-users.

The technical approach is presented in Fig. 1, where the working architecture is illustrated with specific emphasis upon the functional elements and interfaces. The corresponding system is equipped with a two-tier virtualised execution environment, materialised in the form of the Edge DC (Data Centre), which allows also the provision of MEC capabilities to the mobile operators for enhancing the user experience and the agility in the service delivery [16-18].

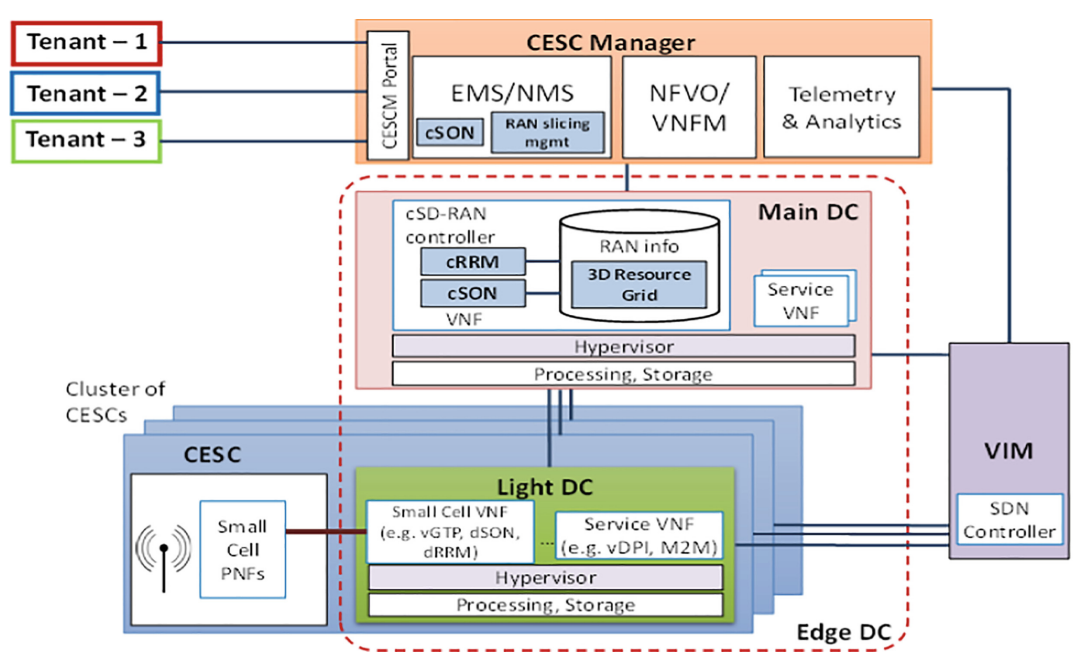

Fig. 1. 5G ESSENCE system architecture.

Both tiers are briefly discussed as follows:

- The first tier, that is the Light DC hosted inside the CESCs, is used to support the execution of VNFs for carrying out the virtualisation of the Small Cell access. In this regard, network functions supporting traffic interception, GTP encapsulation/ decapsulation and some distributed RRM (Radio Resources Management)/SON (Self-Organising Networks) functionalities are expected to be executed therein. VNFs that require low processing power such as, for example, a Deep Packet 
Inspection (DPI), a Machine-to-Machine (M2M) Gateway and so on, could also be hosted here.

- The second cloud tier, that is the Main DC, hosts more computation intensive tasks and processes that need to be centralised in order to have a global view of the underlying infrastructure. This encompasses the cSD-RAN (centralised SoftwareDefined RAN) controller which will be delivered as a VNF running in the Main DC and makes control plane decisions for all the radio elements in the geographical area of the CESC cluster, including the centralised Radio Resource Management (cRRM) over the entire CESC cluster.

Our intended approach demonstrates a combined 5G-based video production and video distribution towards delivering benefits to both media producers and mobile operators, who will be able to offer enriched event experience to their subscribers. The production/distribution of locally generated content through the respective platform, coupled with value-added services and rich user context, can enable secure, highquality and resilient transmission, in real-time and with minimal latency [19].

In the context of the 5G ESSENCE infrastructure sharing support, each network operator will be in position to optimise his network usage, resulting in lower operating expenses (OPEX). Additionally, network operators will be able to rapidly deploy new services, to deliver directly to users higher quality of experience (QoE) and offer "Content as-a-Service", increased bandwidth and storage solutions to content providers and venue owners [9].

The content providers will also benefit from reduced latency and improved user QoE by positioning content on mobile edge and, in addition, they can offer augmented services by leveraging the network information. Finally, the stadium owner will obtain additional benefits by leveraging the deployed dedicated infrastructure and its functionalities, by capitalising live content to spectators from many cameras, statistics, etc.

The developed scenario provides the logic for distributing the live video feeds received from the local production room to local spectators in a highly efficient manner. The Municipal Football Stadium "Stavros Mavrothalassitis" in the city of EgaleoAthens in Greece is covered with a cluster of multitenant, eMBMS-enabled as CESCs and, together with the CESCM (CESC Manager) and the Main DC, they can all potentially be connected to the core networks of multiple telecom operators [20]. The video content from cameras is sent for processing locally at the Edge DC (similar to the proposed use case by ETSI MEC [21]). MEC offers application developers and content providers cloud-computing capabilities and an IT service environment at the edge of the network [22]. This environment is characterized by ultra-low latency and high bandwidth as well as real-time access to radio network information that can be leveraged by applications. MEC provides a new ecosystem and value chain. Operators can open their RAN edge to authorized third-parties, allowing them to flexibly and rapidly deploy innovative applications and services towards mobile subscribers, enterprises and vertical segments. Then the video streams are broadcasted locally using the CESCs and spectators are able to dynamically select between different streams offered. In this and in similar massive event scenarios, the data traffic does not impact the backhaul connection since it is produced, processed and consumed locally (Fig. 2). 


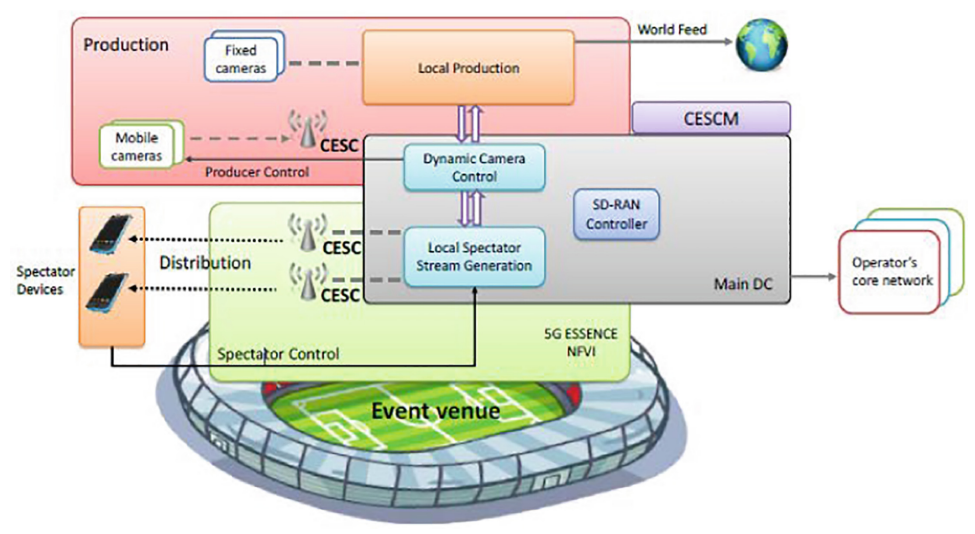

Fig. 2. 5G edge network acceleration in a stadium.

To provide a comprehensive overview of the use case, it is worth highlighting the business roles that can be envisaged. Actors involved in this scenario are listed below:

- Small Cell Network Operator (SCNO): Owner of the infrastructure deployed in the stadium.

- Virtual Small Cell Network Operator (VSCNO): Users of the infrastructure available in the stadium to provide services to the end-users.

- End-Users: Users of the networking services.

- Mobile Operators (MOs): Network operators responsible for bringing the network and communication services to the stadium.

- Service Providers (SPS): Companies providing some of the VNFs to the SCNO. Examples of key service providers for this use case are as follows: (i) Football/sport society; (ii) live event organizer; (iii) municipality, and; (iv) video/content provider.

- Spectrum Owner: In the licensed spectrum case, it is the stadium which leases the spectrum from an operator or a mobile operator that offers a service.

\section{Technical Enablers and Functionalities}

This section intends to present the mapping between the testbed architecture for assessing the respective stadium use case, based on the testbed components presented in previous sub-sections and the corresponding general 5G ESSENCE architecture (as shown in Fig. 1). This architecture serving our stadium use case is illustrated in Fig. 3, and consists of several main elements that are briefly discussed as follows:

- The CESC is composed by a small cell physical network function attached to an execution platform (i.e. micro-server) able to run VNFs corresponding to either small cell functionality or specific service-level functions [23]. 


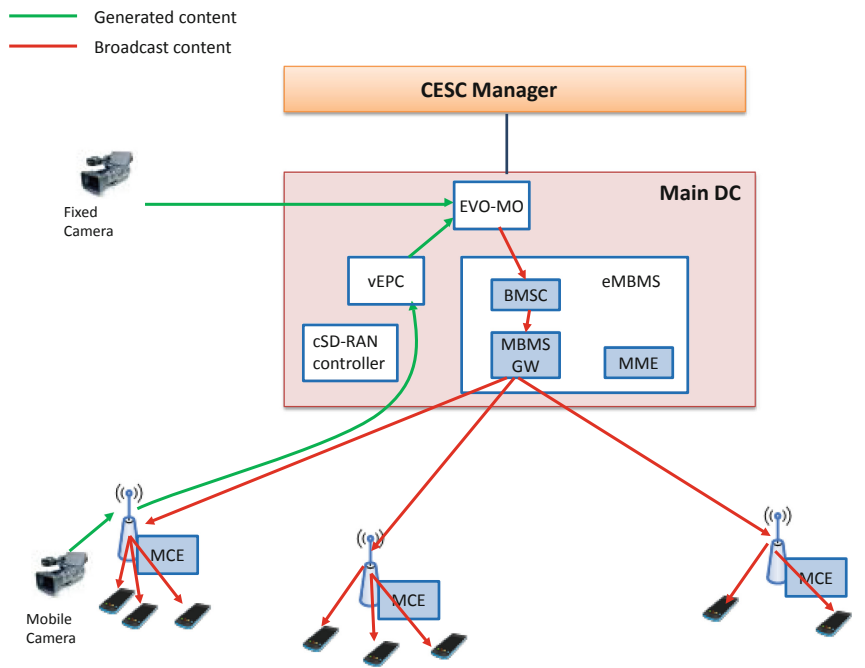

Fig. 3. Stadium demonstration architecture.

- The Edge DC is a two-tier virtualised execution environment composed by the Light DC (resulting from the aggregation of the micro-servers of each CESC) and the Main DC (it is centralised and can host more computation intensive tasks).

- The CESCM includes the components of the ETSI NFV MANO (Management and Orchestration) framework [15], that is the NFVO (NFV Orchestrator) and VNF Manager (VNFM) for carrying out the lifecycle management of network services and VNFs, the Element Management System (EMS)/Network Management System (NMS) for carrying out the management of the deployed CESCs in terms of Fault, Configuration, Accounting, Performance, Security (FCAPS) operations, and a telemetry and analytics module that collects and analyses relevant indicators of the network operation [24].

- The Virtualised Infrastructure Manager (VIM) manages, monitors and optimises the operation of the NFVI (NFV Infrastructure) resources.

The support of eMBMS in this relies upon several functionalities hosted at different components of the original architecture. At the CESC side, the small cells should incorporate the functionality associated to the Multi-Cell/Multicast Coordination Entity (MCE) that manages the allocation of resources for eMBMS transmissions [25]. In turn, the Main DC hosts the rest of components of the eMBMS architecture in the form of a VNF that includes the BMSC (Broadcast Multicast Service Center), the MBMS (Multimedia Broadcast/Multicast Service) GW (Gateway) [26, 27] and the MME (Mobility Management Entity). The video content to be delivered to the eMBMScapable UEs will be produced by the Edge Video Orchestrator - Master Orchestrator (EVO-MO) that will also run as a VNF in the Main DC [28]. In the selected stadium use case, the EVO-MO does not perform CPU (Central Processing Unit) intensive tasks (such as transcoding for external video sinks) and, thus, a simple DC will have enough capacity. 
At the same time, this choice ensures that the video content is confined within the area covered by the small cell. The Main DC also incorporates a virtual EPC (Evolved Packet Core) function (the EPC is the latest evolution of the 3GPP core network architecture) that allows the video upstreaming and offloading the traffic locally in the stadium, where the CESCs have been deployed without the need to have access to the backbone of the operator [29].

At the CESCM side, Fig. 1 illustrates the EMS/NMS, which is in charge of configuring the parameters of the small cells, and the NFVO/VNFM components, in charge of, for example, instantiating and terminating the abovementioned VNFs. In Fig. 3 we illustrate the interrelationships between the different components of the architecture for demonstrating the delivery of eMBMS data to the User Equipments (UEs). The demonstration assumes that the eMBMS content can be originated either from fixed cameras or from mobile cameras hosted at different UEs. In both cases the images captured by the cameras are delivered to the EVO-MO in upstream via the virtual EPC (vEPC) for processing it. For that purpose, mobile cameras will have access to the CESCs as other UEs and will transmit information through the radio interface by means of a data radio bearer. At the main DC, this data will be received by the local vEPC who will deliver it to the EVO-MO. Based on the data received from the cameras, the EVO-MO will produce the content to be distributed to the UEs [30]. As seen in Fig. 3, this content is injected to the BMSC inside the eMBMS VNF and then it is passed to the MBMS GW who delivers it to the involved small cells.

\section{Pilot Description}

The selected facility is covered with a cluster of multitenant, eMBMS-enabled CESCs and, together with the CESCM and the Main DC, can be connected to the core networks of several participating telecom operators. The video content from cameras is sent for processing locally at the Edge DC. The video streams are broadcasted locally by using the CESCs. Spectators are able to dynamically select between different offered broadcast streams. The main infrastructure needed for this demo is depicted as in Fig. 4, below:

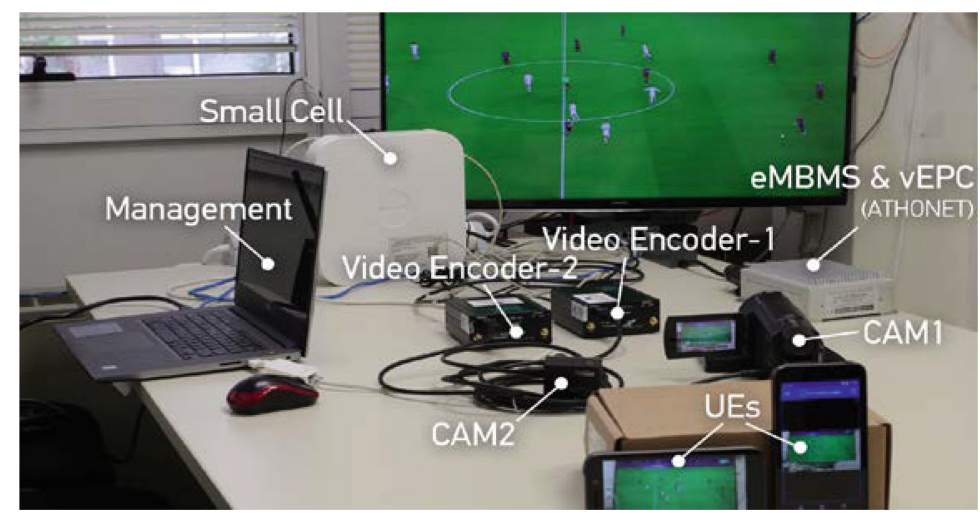

Fig. 4. Main infrastructure of the realization of the use case. 
Following to the successful integration and testing of this use case infrastructure, the following network services have been deployed:

- Multicast Video Delivery in Multi/Single View: Here the intended objectives have been to assure that: (i) the multicast video is delivered to the small cell and UEs, by receiving related multicast streams; (ii) multicast video is delivered to UEs and can be watched in Multi/Single View (in this case the aim has been to confirm that users can watch videos delivered by multicast in single view and multi-view with the quality known from unicast transmission); (iii) the small cell provides sufficient capacity to all available multicast videos; (iv) stable delivery of multicast to UEs is feasible; in particular, no significant performance degradation observed during evaluation period; (v) the multicast delivery effectively covers all areas of interest in the stadium, and; (vi) audio/video synchronization is achieved for multicast delivery.

- UE View Switching during the Video Delivery: In this service the intended objective has been to assure that quality of experience is preserved when switching from multi-view to single view and vice versa; in fact, relevant switching times have been small and do not affect user experience significantly.

- Video Delivery with Handover: In the context of this service the intended objectives have been to assure that: (i) seamless handover for multicast video transmission takes place; in fact, no video degradation observed when moving from one cell to another, and; (ii) seamless multicast video transmission for mobile encoder is feasible; in particular, no difference in unicast and multicast delivery observed when the video source moves from cell to cell.

- Unicast vs. Multicast Video Delivery: In this service case, the intended objectives have been to: (i) demonstrate the reduction in resource usage when using multicast delivery; in fact, significant reductions in resource usage (radio, backhaul) have been performed when using multicast instead of unicast, and; (ii) compare transmission delay in unicast and multicast video delivery; here results have demonstrated that the transmission delay for multicast delivery is very slightly higher compared to unicast transmission (due to the longer chain of entities crossed) but not affecting the user experience.

Two successful demos already took place in the stadium. The first demo took place in July 2019, during Egaleo team's training. The second one took place in October 2019, where two football teams had a typical 90-min match. The event was broadcasted via the $360^{\circ}$ camera to the eMBMS-enabled end-devices of the viewers. Apart from the 5G ESSENCE partners, many external viewers also attended this event, including two members of the Greek Parliament, the Mayor of Egaleo with two his predecessors, as well as the Deputy Minister of Digital Governance.

\section{Market Projection}

5G will undoubtedly change our everyday lives and the world as a whole. It will have many political, social and economic consequences. New applications and use cases will emerge, which will allow not only for the operators to expand their businesses, but also 
for new players to enter the market [31]. In the $5 \mathrm{G}$ era, different industry verticals are looking for leveraging the influence of modern technologies to boost productivity across paths of the economy [32]. The main idea of the present use case has been to overcome the "hiccups" while at a stadium, including missing parts of the actual game due to different factors, as well as not receiving a good signal while attending an event in a limited space with over several thousands other people. This use case has become able to demonstrate the broadcast capabilities of LTE/5G networks for video processing at the edge by reducing the backhaul traffic to the macro network by leveraging SCs which are connected to a local EPC. The use of the EPC expands the coverage and capacity and paves the way to multimedia transmission to tackle viewing problems audiences' in a stadium face [33]. This is done by multi-angled streaming which will be offered to the audience as a service to improve the QoE.

Considering that the majority of attendees at live events check regularly their phones during the event, there is enough space for introduction of this product. The estimated price that will be charged for the service is 65.00 Euros per year per user, which can be considered a fairly low-price which will leave the business owners a good margin for profit. We take an assumption that the stadium where the respective service will be installed has average match attendance of 48,000 people (which is around the average spectator number in Bundesliga in Germany), out of whom $10 \%$ will be using our service on year 1.

That $10 \%$ is a very conservative estimation considering the following facts [34]: (i) $18 \%$ of overall fans check their phones at matches; (ii) $31 \%$ of millennials check their phones during a live event; (iii) $80 \%$ of all age groups use a second screen to check scores in other matches, to get expert analysis or to engage with others online over the match; (iv) $50 \%$ of the attendees believe that virtual view would improve experience, and; (v) $63 \%$ of the attendees believe that video replay would generate excitable experience.

Then we assess that an additional $3 \%$ increase of users per year will occur, and each small cell can support up to 400 simultaneous active subscribers; thus for 4,800 users we will be requiring 14 small cells but with a $3 \%$ increase per year in a 10 years period we will be needing a total of 16 small cells for 6,263 users. Further parameters in our calculation have been:

- 25 events per year per stadium (i.e.: in Bundesliga 17 matches per year +7 for cup +8 for European events).

- Energy consumption is estimated as 1,000.00 Euros per year since the infrastructure will be active only $3 \mathrm{~h}$ per event (pre-match and after match along with actual game time reaching a total of $25 \times 3=75 \mathrm{~h}$ of use per year) including small cells, cameras, backhaul, router and server energy consumption.

- Tax rate $=29.8 \%$ [35].

- WACC (Weighted Average Cost of Capital) $=7.45 \%$ considering that the project on this scenario will be co-financed on a 50-50 basis by the involved 5G ESSENCE network operators (i.e.: OTE S.A. (Greece) and Wind TRE SpA (Italy)).

Taking into consideration the sales forecast and the cost estimation, and assessing the NPV (net present value) as 300,190.00 Euros, the payback period as is in year 4 and the IRR (internal rate of return) as $14.33 \%$, we can conclude that the initial investment 
required is 516,340.00 Euros including 469,400.00 Euros for the CAPEX and 46,940.00 Euros for OPEX costs.

\section{Conclusion}

The 5G ESSENCE project demonstrates a combined 5G-based video production and video distribution towards delivering benefits to both media producers and mobile operators, who will be able to offer enriched event experience to their subscribers. The production/distribution of locally generated content through the related platform, coupled with value-added services and rich user context, enables secure, high-quality and resilient transmission in real-time, thus ensuring minimal latency. This delivers benefits to media producers and mobile operators as it allows them offering a highly interactive fan experience and optimises operations by deploying key functionalities at the edge (i.e., eMBMS or local network services like real-time analytics together with multitenancy support by small cells).

A large-scale facility in a municipality stadium was used for the validation of the respective use case. The coverage in this facility was provided by a cluster of multitenant, eMBMS-enabled SCs and a main DC able to be connected to the core networks of multiple telecom operators. The Edge DC is processing video content from cameras deployed on-site, which is broadcasted locally without affecting the backhaul. In order to "address" the needs and requirements of a robust and agile network management, and building upon the pillars of network functions virtualisation, mobile-edge computing and cognitive management, our attempt aimed to deliver new business models and revenue streams in the real-life use cases associated to the vertical industry of entertainment, which is purely the case of edge network acceleration in a crowded event. This "opens the door" to venue owners (e.g., municipalities, stadiums, site owners and virtually anyone who manages a property and can install and run a local Small Cell network) to deploy a low cost infrastructure and to act as neutral host network and service provider. Although probably none of such entities would offer static network coverage, many of them could foresee adequate chances for profits generated by exploiting the 5G ESSENCE concepts of multitenant small cells, able to provide wireless network coverage coupled with added value services in close proximity to customers and visitors that belong to multiple network operators and vertical industries.

Acknowledgments. This work has been performed in the joint scope of the $5 G$ ESSENCE and the MOTOR5G European Research Projects and has been supported by the Commission of the European Communities/H2020 under Grant Agreements No. 761592 and No. 861219, respectively.

\section{References}

1. Andrews, J.G., Buzzi, S., Choi, W., Hanly, S.V., et al.: What will 5G be. IEEE JSAC. 32(6), 1065-1082 (2014). Special Issue on 5G Wireless Communications Systems 
2. Chochliouros, I.P., et al.: Challenges for defining opportunities for growth in the $5 \mathrm{G}$ era: the SESAME conceptual model. In: Proceedings of the 25th European Conference on Networks and Communications (EuCNC), pp. 1-5 (2016)

3. Chochliouros, I.P., et al.: Putting intelligence in the network edge through NFV and cloud computing: the SESAME approach. In: Boracchi, G., Iliadis, L., Jayne, C., Likas, A. (eds.) EANN 2017. CCIS, vol. 744, pp. 704-715. Springer, Cham (2017). https://doi.org/10.1007/ 978-3-319-65172-9_59

4. Weldon, M.K.: The Future X Network: A Bell Labs Perspective. CRC Press, Boca Raton (2016)

5. Chochliouros, I.P., et al.: Business and market perspectives in 5G Networks. In: Proceedings of the Joint 13th CTTE and 10th CMI Conference 2017, pp. 1-6. IEEE (2017)

6. 5G ESSENCE H2020/5G-PPP Project (GA No. 761592). http://www.5g-essenceh2020.eu

7. Chochliouros, I.P., et al.: A model for an innovative 5G-oriented architecture, based on small cells coordination for multi-tenancy and edge services. In: Iliadis, L., Maglogiannis, I. (eds.) AIAI 2016. IFIP AICT, vol. 475, pp. 666-675. Springer, Cham (2016). https://doi.org/10. 1007/978-3-319-44944-9_59

8. Goratti, L., et al.: Network architecture and essential features for 5G: the SESAME project approach. In: Iliadis, L., Maglogiannis, I. (eds.) AIAI 2016. IFIP AICT, vol. 475, pp. 676685. Springer, Cham (2016). https://doi.org/10.1007/978-3-319-44944-9_60

9. Kostopoulos, A., et al.: Use cases for $5 \mathrm{G}$ networks using small cells. In: Iliadis, L., Maglogiannis, I., Plagianakos, V. (eds.) AIAI 2018. IFIP AICT, vol. 520, pp. 39-49. Springer, Cham (2018). https://doi.org/10.1007/978-3-319-92016-0_4

10. Costa-Perez, X., Swetina, J., Guo, T., Mahindra, R., Rangarajan, S.: Radio access network virtualization for future mobile carrier networks. IEEE Commun. Mag. 51(7), 27-35 (2013)

11. Fajardo, J.O., et al.: Introducing mobile edge computing capabilities through distributed 5G Cloud enabled small cells. Mobile Netw. Appl. 21(4), 564-574 (2016). https://doi.org/10. 1007/s11036-016-0752-2

12. Afolabi, I., Taleb, T., Samdanis, K., Ksentini, A., Flinck, H.: Network slicing and softwarization: a survey on principles, enabling technologies, and solutions. IEEE Commun. Surv. Tutor. 20(3), 2429-2453 (2018)

13. Sallent, O., Pérez-Romero, J., Ferrús, R., Augusti, R.: On radio access network slicing from a radio resource management perspective. IEEE Wirel. Commun. J. 24(5), 166-174 (2017)

14. The 3rd Generation Partnership Project (3GPP): 3GPP TR 32.851 V12.2.0: Telecommunications management; Study on operations, administration and management (OAM) aspects on network sharing (Release 12) (2014)

15. European Telecommunications Standards Institute (ETSI): NFV Management and Orchestration - An Overview, GS NFV-MAN 001 v1.1.1. ETSI (2014)

16. Chochliouros, I.P., et al.: Enhancing network management via NFV, MEC, cloud computing and cognitive features: the "5G ESSENCE" modern architectural approach. In: Iliadis, L., Maglogiannis, I., Plagianakos, V. (eds.) AIAI 2018. IFIP AICT, vol. 520, pp. 50-61. Springer, Cham (2018). https://doi.org/10.1007/978-3-319-92016-0_5

17. Chochliouros, I.P., Kostopoulos, A., Spiliopoulou, A.S., Kourtis, A., et al.: Small cells, $\mathrm{NFV}$, and cloud computing as enablers for offering innovative $5 \mathrm{G}$ services: from the SESAME to the 5G ESSENCE architectural framework. In: Proceedings of the 27th European Conference on Networks and Communications (EuCNC), pp. 570-574. IEEE (2018)

18. Chochliouros, I.P., Giannoulakis, I., Spiliopoulou. A.S., et al.: A novel architectural concept for enhanced 5G network facilities. In: MATEC Web of Conferences, CSCC-2017, vol. 125, no. 03012, pp. 1-7 (2017) 
19. Fajardo, J.O., Taboada, Y., Liberal, F.: Improving content delivery efficiency through multilayer mobile edge adaptation. IEEE Netw. Manag. 29(6), 40-46 (2015)

20. Giannoulakis, I., Kafetzakis, E., Trajkovska, I., et al.: The emergence of operator-neutral small cells as a strong case for cloud-like computing at the mobile edge. Trans. Emerg. Telecommun. Technol. 27(9), 1152-1159 (2016)

21. European Telecommunication Standards Institute (ETSI): Multi-Access Edge Computing. http://www.etsi.org/technologies-clusters/technologies/multi-access-edge-computing

22. Kostopoulos, A., Chochliouros, I.P., Giannoulakis, I., Kourtis, A., Kafetzakis, E.: Small cellsas-a-service in 5G networks. In: Proceedings of the IEEE 2018 International Symposium on Broadband Multimedia Systems and Broadcasting (BMSB), pp. 1-4. IEEE (2018)

23. Kostopoulos, A., Chochliouros, I.P., et al.: Network functions for supporting 5G services. In: Proceedings of the 28th European Conference on Networks and Communications (EuCNC), pp. 1-4. IEEE (2019)

24. Chochliouros, I.P., et al.: Using small cells for enhancing 5G networks. In: Proceedings of the IEEE 2017 Conference on Network Function Virtualization and Software Defined Networks (NFV-SDN), pp. 1-6. IEEE (2017)

25. The 3rd Generation Partnership Project (3GPP): TS 23.246 v13.3.0: Multimedia Broadcast/Multicast Service (MBMS); Architecture and functional description (2015)

26. The 3rd Generation Partnership Project (3GPP): TS 22.146 V15.0.0: Technical Specification Group Services and System Aspects; Multimedia Broadcast/Multicast Service (MBMS); Stage 1 (Release 15) (2019)

27. The 3rd Generation Partnership Project (3GPP): TS 22.246 V15.0.0: Technical Specification Group Services and System Aspects; Multimedia Broadcast/Multicast Service (MBMS) user services; Stage 1 (Release 15) (2018)

28. Smart Mobile Labs AG: Edge Video Orchestration EVO - Product description, Release 2.1 (2018)

29. Kostopoulos, A., Chochliouros, I.P., Sfakianakis, E., Munaretto, D., Keuker, C.: Deploying a 5G architecture for crowd events. In: Proceedings of the 2019 IEEE International Conference on Communications (ICC) Workshops, pp. 1-6. IEEE (2019)

30. Kostopoulos, A., Chochliouros, I.P., Sfakianakis, E., Munaretto, D., Keuker, C.: A cloudbased architecture for video services in crowd events. In: MacIntyre, J., Maglogiannis, I., Iliadis, L., Pimenidis, E. (eds.) AIAI 2019. IFIP AICT, vol. 560, pp. 7-18. Springer, Cham (2019). https://doi.org/10.1007/978-3-030-19909-8_1

31. Mordor Intelligence: 5G Infrastructure Market - Growth, Trends and Forecast (2020-2025). Mordor Intelligence (2020)

32. Kostopoulos, A., Chochliouros, I.P., Spada, M.R.: Business challenges for service provisioning in 5G networks. In: Abramowicz, W., Corchuelo, R. (eds.) BIS 2019. LNBIP, vol. 353, pp. 423-434. Springer, Cham (2019). https://doi.org/10.1007/978-3-030-20485-3_ 33

33. Kostopoulos, A., Chochliouros, I.P., Sfakianakis, E., Munaretto, D., Keuker, C., Giannoulakis, I.: 5G edge network acceleration for crowd events. In: Proceedings of the 19th International Conference WWW/Internet, ICWI 2019, pp. 117-124. IADIS (2019)

34. McIntyre, H.: Millennials, Live Events, and Smartphones: A Look Into Their Behavior. Hollywood \& Entertainment (2015). https://www.forbes.com/sites/hughmcintyre/2015/05/ 07/millennials-live-events-and-smartphones-a-look-into-their-behavior/\#2b7ea5623e79

35. Asen, E.: Corporate Income Tax Rates in Europe. Tax Foundation (2019). https:// taxfoundation.org/corporate-tax-rates-europe-2019/ 